randomized controlled trials examining the impact of hydroxychloroquine (HCQ) on SLE were men. Thus, our objective was to examine the disease activity of both women and men with SLE treated with HCQ. In a cross-sectional analysis, we hypothesized that men with SLE would have higher disease activity than women based on average SLE Disease Activity Index (SLEDAI) 2K and Damage Index (DI) scores.

Methods Patient information was collected from the Canadian Network for Improved Outcomes in SLE (CaNIOS) database. All patients had an SLE diagnosis confirmed using the American College of Rheumatology (ACR) criteria. Inclusion in this study required that patients be $\geq 18$ years of age and be currently taking any prescribed dosage regimen of HCQ. Data for these analyses were collected at entry into CaNIOS. Descriptive characteristics included health habits, ethnicity, immunosuppressant medication usage and SLEDAI 2K 10 day and DI scores. Sex differences in SLEDAI $2 \mathrm{~K}$ and DI scores were analyzed using multivariate regression analyses controlling for prednisone use, current age, age at SLE onset and disease duration.

Results A total of 499 patients ( $n=57$ men, $n=442$ women) treated with HCQ were included (table 1). Men appeared to have slightly lower SLEDAI $2 \mathrm{~K}$ scores and higher DI scores than women, and had slightly higher use of prednisone. However, regression analyses revealed no differences in SLEDAI $2 \mathrm{~K}$ scores between sexes, yet current age was significant $(\beta=0.228, p=0.021)$. DI scores were significantly associated with current age $(\beta=0.349, p=0.001)$ and disease duration $(\beta=0.170, p=0.002)$ but not sex.

Conclusions In contrast to our hypothesis, we found no sex differences in disease activity or damage index between men and women. Results of this study are limited by lack of control for HCQ dose and duration of use which will be addressed in future analyses. Future analyses will investigate potential sex differences in longitudinal changes in SLEDAI and DI scores in response to $\geq 3$ months of HCQ therapy.

\section{CS-26 WHAT DO WE MEAN WHEN WE SAY SLE?}

${ }^{1}$ Linjia Jia, ${ }^{2}$ Ecem Sevim, ${ }^{2}$ Medha Barbhaiya, ${ }^{2}$ Michael D Lockshin*. 'Weill-Cornell Medicine, New York, New York, USA; ${ }^{2}$ Barbara Volcker Center, Hospital for Special Surgery, New York, New York, USA

\subsection{6/lupus-2018-Ism.61}

Background Although lupus spectrum illness contains at least four variants (antibody only, undifferentiated autoimmune disease, typical SLE, and overlap SLE), clinical, epidemiological, and basic science publications do not use distinguish among these groups when they speak of SLE, thereby muddling interpretation of the results.

Methods We systematically reviewed clinical, epidemiological, and basic science publications from the last 5 years to determine definitions they used for SLE.

Results Of 366 papers on human SLE, 197 were suitable for analysis. Of these, 32 were basic/translational, 151 clinical trials and studies, and 14 epidemiological. Between $66 \%$ and $79 \%$ of papers specified or implied use of ACR criteria, of which $0 \%-16 \%$ also required presence of anti-DNA or antiSm antibody. 19\%-29\% of papers specified exclusion criteria, of which 10\%-14\% specifically excluded patients with overlap disease. Disease activity was considered in $14 \%-22 \%$ and stable disease was required in 7\%-14\% of papers. $0 \%-3 \%$ of papers considered disease duration (table 1).
Of 132 studies on mouse SLE, 24\% use NZB variants, $36 \%$ MRL-lpr variants and 39\% used variants/induced illness in $\mathrm{C} 57 \mathrm{Bl} / 6 \mathrm{~J}$ mice. Definition of SLE required presence of anti-DNA antibody in $47 \%$ of studies, ANA in 10\%, proteinuria in $39 \%$ and immunohistochemistry (usually renal) in $57 \%$. $15 \%$ of papers defined disease onset $\leq 8$ weeks, $15 \% 8-16$ weeks, and $57 \%$ did not indicate time of disease onset. The fact that the paper spoke of animal SLE was indicated in the titles of $46 \%$ of papers.

\begin{tabular}{lllll}
\multicolumn{1}{l}{ Abstract CS-26 Table } & \multicolumn{5}{l}{} \\
\hline Type of study (N) & Basic (32) & Clinical (151) & Epidem (14) & All (197) \\
\hline ACR Criteria only & 69 & 66 & 79 & 67 \\
aDNA or aSm specified & 13 & 9 & 0 & 9 \\
Exclusion criteria specified & 19 & 31 & 29 & 29 \\
Exclusion overlap specified & 13 & 10 & 14 & 11 \\
Activity SLEDAI or BILAG & 22 & 16 & 14 & 17 \\
Stable disease specified & 13 & 7 & 14 & 9 \\
Duration specified & 0 & 3 & 0 & 3 \\
\hline
\end{tabular}

Criteria used in 197 studies on human SLE, indicating criteria for diagnosis, inclusions, exclusions, serology, activity, and disease duration.

Conclusion Publications on SLE use a wide variety of definitions, inclusion, and exclusion criteria and often use the term SLE without specifying animal or human. Consensus definitions and stratification rules are necessary to obtain greater homogeneity in studies of SLE. (We will propose methods by which greater consensus can be obtained.)

Acknowledgements Supported by the Barbara Volcker Center and the Weill Cornell Medicine AOC program.

\section{CS-27 MORTALITY AMONG PATIENTS WITH SYSTEMIC LUPUS ERYTHEMATOSUS HOSPITALIZED WITH SEPSIS VARIES WIDELY AMONG U.S. HOSPITALS}

${ }^{1}$ Michael M Ward*, ${ }^{2}$ Maria Tektonidou, ${ }^{1}$ Abhijit Dasgupta. ${ }^{1}$ National Institute of Arthritis and Musculoskeletal and Skin Diseases, NIH, U.S; ${ }^{2}$ First Department of Propaedeutic Internal Medicine, Joint Academic Rheumatology Program, Medical School, National and Kapodistrian University of Athens, Athens, Greece

\subsection{6/lupus-2018-Ism.62}

Background Sepsis is a serious infection that is often difficult to treat. We investigated if the risk of mortality in patients with systemic lupus erythematosus (SLE) hospitalized with sepsis varies among hospitals.

Methods We used the National Inpatient Sample (2002-2011) to obtain data on outcomes of adults age 18 to 64 with SLE who were admitted with a diagnosis of sepsis. We included 424 hospitals that had at least 5 hospitalizations of patients with SLE and sepsis. We abstracted data on demographic features, diagnoses, and mortality for these patients and for patients without SLE hospitalized with sepsis at the same set of hospitals. We used machine learning methods to derive the expected risk of in-hospital mortality for each patient, based on demographic features, insurance status, comorbidities, and diagnoses related to organ failure. We then computed the observed/expected ratio for in-hospital mortality separately for SLE and non-SLE patients by hospital. To further adjust for case-mix, the ratio of these two ratios (RR) was used to 
evaluate the relative likelihood of death among those with SLE compared to those without SLE at the same hospital.

Results We included 4001 hospitalizations of patients with SLE and sepsis, and 202888 hospitalizations of patients with sepsis without SLE. The number of SLE hospitalizations ranged from 5 to 66 per hospital. Across all hospitals, 11.5\% of patients with SLE and $13.1 \%$ of patients without SLE died during the hospitalization. The RR was greater than 1.0 in $44 \%$ of hospitals, and greater than 2.0 in $16 \%$ of hospitals, indicating increased risk of mortality by a factor of two or more among patients with SLE compared to those without SLE at the same hospital. Based on classification tree analysis, large urban hospitals were less likely to have $\mathrm{RR}>2$ than smaller hospitals (13\% vs 22\%), particularly those in the midwest or northeast (35\%).

Conclusions Risks of mortality due to sepsis among patients with SLE varied widely among U.S. hospitals. Risks were higher for patients with SLE at smaller hospitals.

Acknowledgements Supported by the Intramural Research Program, NIAMS/NIH.

\section{CS-28 VALIDATION OF REMISSION AND LUPUS LOW DISEASE ACTIVITY STATEAS PREDICTORS OF ORGAN DAMAGE IN SLE}

${ }^{1}$ Michelle Petri ${ }^{*},{ }^{1}$ Daniel Goldman, ${ }^{2}$ Laurence S Magder. 'Johns Hopkins University School of Medicine, Baltimore, MD, USA; ${ }^{2}$ University of Maryland, Baltimore, MD USA

10.1136/lupus-2018-Ism.63
Background Outcome measures that combine control of SLE activity and prednisone reduction are clinically relevant. A clinical goal in SLE is to reduce risk of long-term organ damage. We assessed whether two recently proposed disease activity outcomes were predictive of future damage.

Methods For each month of follow-up in a large SLE cohort, we determined whether the patient was in Clinical Remission (as defined by the DORIS working group) or lupus low disease activity state (LLDAS) (as defined by Franklyn et al). Clinical Remission was defined as a PGA $<0.5$, clinical SLEDAI $=0$ and no prednisone or immunosuppressants. Clinical Remission on Treatment allowed for prednisone $\leq 5 \mathrm{mg} /$ day and immunosuppressant use. LLDAS was defined as a SLEDAI $\leq 4$, PGA $\leq 1.0$, no major organ activity, and no new activity. LLDAS on treatment allowed for prednisone use $\leq 7.5 \mathrm{mg} / \mathrm{d}$ and immunosuppressants. Damage was defined using the SLICC/ACR Damage index.

Results There were 81118 person-months observed among 2026 patients $(92 \%$ female, 53\% Caucasian, 39\% AfricanAmerican). Table 1 shows the rates of damage, per person month, in subgroups defined by Remission or LLDAS.

Damage rates were relatively low when LLDAS was achieved at least $50 \%$ of the time. These rates were similar to those experienced by patients who met a more stringent treatment restriction with Remission on Treatment at least $50 \%$ of the time.

Conclusions Percent time in LLDAS had a clear dose response for rate ratios of organ damage. Even 25\% of time in

Abstract CS-28 Table 1 Rates of new damage, in subgroups defined by past levels of disease activity

\begin{tabular}{|c|c|c|c|c|c|}
\hline Percentage of Prior Months in: & $\begin{array}{l}\text { Number of person- } \\
\text { months observed }\end{array}$ & $\begin{array}{l}\text { Number of months } \\
\text { with an increase in } \\
\text { SLICC/ACR Damage } \\
\text { Index }\end{array}$ & $\begin{array}{l}\text { Rate of damage } \\
\text { per } 100 \text { person } \\
\text { months }\end{array}$ & Rate Ratios & P-values \\
\hline Clinical Remission & - & - & - & - & - \\
\hline None & 35772 & 406 & 1.13 & 1.0 (Ref) & - \\
\hline Not none, but $<25 \%$ & 14358 & 102 & 0.71 & $0.60(0.48,0.75)$ & $<0.0001$ \\
\hline $25 \%$ to $50 \%$ & 6573 & 50 & 0.76 & $0.66(0.46,0.94)$ & 0.023 \\
\hline $50 \%$ to $75 \%$ & 3845 & 27 & 0.70 & $0.63(0.42,0.97)$ & 0.035 \\
\hline $75 \%+$ & 1641 & 10 & 0.61 & $0.58(0.30,1.15)$ & 0.12 \\
\hline Clinical Remission on Treatment & - & - & - & - & - \\
\hline None & 16491 & 250 & 1.52 & 1.0 (Ref) & - \\
\hline Not none, but<25\% & 20169 & 170 & 0.84 & $0.54(0.44,0.67)$ & $<0.0001$ \\
\hline $25 \%$ to $50 \%$ & 14344 & 103 & 0.72 & $0.46(0.36,0.60)$ & $<0.0001$ \\
\hline $50 \%$ to $75 \%$ & 8396 & 54 & 0.64 & $0.43(0.30,0.60)$ & $<0.0001$ \\
\hline $75 \%+$ & 2789 & 18 & 0.65 & $0.45(0.27,0.75)$ & 0.0019 \\
\hline LLDAS & - & - & - & - & - \\
\hline None & 30366 & 343 & 1.13 & 1.0 (Ref) & - \\
\hline Not none, but<25\% & 10880 & 106 & 0.97 & $0.86(0.69,1.07)$ & 0.18 \\
\hline $25 \%$ to $50 \%$ & 5012 & 40 & 0.80 & $0.70(0.51,0.98)$ & 0.037 \\
\hline $50 \%$ to $75 \%$ & 8494 & 60 & 0.71 & $0.63(0.48,0.83)$ & 0.0010 \\
\hline $75 \%+$ & 7527 & 46 & 0.61 & $0.54(0.40,0.73)$ & $<0.0001$ \\
\hline LLDAS on Treatment & - & - & - & - & - \\
\hline None & 7656 & 117 & 1.53 & 1.0 (Ref) & - \\
\hline Not none, but $<25 \%$ & 10555 & 134 & 1.27 & $0.83(0.65,1.06)$ & 0.14 \\
\hline $25 \%$ to $50 \%$ & 12686 & 129 & 1.02 & $0.66(0.51,0.85)$ & 0.0013 \\
\hline $50 \%$ to $75 \%$ & 18151 & 133 & 0.73 & $0.48(0.37,0.61)$ & 0.0010 \\
\hline $75 \%+$ & 13141 & 82 & 0.62 & $0.40(0.30,0.54)$ & $<0.0001$ \\
\hline
\end{tabular}

